\title{
Practical Evaluation of Automated Measuring System for Grain Size
}

\author{
Yoshinao MISHIMA
}

Research Laboratory of Precision Machinery and Electronics, Tokyo Institute of Technology, Nagatsuta, Midori-ku, Yokohama, Kanagawaken, 227 Japan.

(Received on January 20, 1990; accepted in the final form on April 20, 1990)

\begin{abstract}
The outline of the software for the grain size measurement developed by the Grain Size Measurement Group in the Committee on Materials Evaluation by Image Analysis Basic Research Association on Specific Subjects sponsored by the Iron and Steel Institute of Japan is described. It consists of three parts being binarization of the input image and grain boundary thinning, repair of the grain boundary, and the measurement and its statistical treatment of grain size. The emphasis is put on the first two processes and the effect of changing the default parameters for binarization and thinning processes on the result of the following repair process is discussed. Then the effect of sample preparation condition on the results of these processes is examined using a common specimen but having different states of polishing and etching. It is shown that for such specimens as polished to $3 \mu \mathrm{m}$ or finer diamond paste the repair process can be successfully done to give a grain network which well represents the original image. It is found that the variation in etching conditions hereby examined less affects the results. Through these discussions practical usefulness and limitations of the software are visualized.
\end{abstract}

KEY WORDS: image analysis; grain size; metallography; personal computer; sample preparation; polishing; binarization.

\section{Introduction}

A number of image analysis systems have become commercially available along with the recent rapid advance in both processing speed and memory capacity of the personal computers at even reduced prices. The commercial image analysis systems are generally multi-purpose and are designed to be capable of such analyses as particle size characterization, grain size measurement and various types of image processing. In most cases, however, the algorithm for any type of analysis is different from system to system.

The Grain Size Measurement (GSM) Group in the Committee on Materials Evaluation by Image Analysis, Basic Research Association on Specific Subjects sponsored by The Iron and Steel Institute of Japan during the period from March, 1986 through February, 1989 has attempted a collaborated research on how consistent the results would be if we carry out grain size measurement of a common specimen using different types of commercial image analysis system. The result was surprising because there was absolutely no agreement among over 5 different systems examined. It was the time when the GSM Group had decided to develop its own image analysis system only for the purpose of grain size measurement. The goal was initially set as follows;

(1) the grain size measurement can be done either from direct $\mathrm{TV}$ image or photographic image of a grain network,

(2) it can be done for a grain network which is the simplest from a metallographic point of view, namely such as ferritic grains without any substructures within them, and
(3) the whole processing should be done within a reasonable time frame such as 10 to $30 \mathrm{~min}$ for a specimen using a personal computer being typically one of the NEG PC-9800 series computers.

The final version of the software for grain size measurement has now become available. It consists of three parts:

1) binarization of an input image and thinning of grain boundary,

2) repair of grain network, and

3) measuring and statistical calculations on grain size characteristics.

Upon operation one can conduct these three parts sequentially with necessary pause and print out at the end of each step. Explanations on the principle and algorithm of each part are made elsewhere in this volume. ${ }^{1-3)}$ When practically using the software, one of the most important parameter to affect the results is the quality of the input image since the software has been developed for relatively simple grain networks as an input. The first process to thin the grain boundaries therefore provides optional parameters for a user to modify the default values for his particular purpose. Here, the effect of changing default parameters on the result for grain boundary thinning and subsequent repair process is first discussed. Then the effect of input image qualities as affected by the preparation techniques of metallographic samples will be examined on the results also of the first and the second parts of the software. To be noted is that the repair process is automatic and has no options for users and the parameter change hereby to be discussed is only for the first part of grain network construction. The statistical analysis on the grain size measurement after the processes discussed here is found elsewhere 
in this volume. ${ }^{3)}$ Finally a comment will be presented upon the limitations of the software in terms of the complexity of the input image. They are of practical importance in utilizing the software developed by the GSM Group.

\section{User Options for Parameter Setting in Bina- rization and Thinning Processes}

\subsection{Steps of Operation and Variable Parameters}

A regular optical micrograph of a grain structure is the first input image taken in by a TV camera, which of course can be done with or without photographic processing. The first step for the computer to work on is the binarization of the input image which is followed by thinning of the binarized image to construct a grain network consisting of a single pixel. A user has two options to go through these processes by designating either "STARTUP1" or "STARTUP2" as a batch file for this purpose being called as "STARTUP" on the current MS-DOS (registered trade mark for Microsoft Disk Operating System). The "STARTUP2" is a simplified version of the "STARTUP1" mainly for the purpose of time-saving.

The major parameters which could be adjusted by users on the "STARTUP" are the followings. Regarding the principle and physical meaning of each step, consult with the article by Sakaue in this volume. ${ }^{1)}$

(1) Setting of a Threshold for Binarization of the Input Image

Default setting here is [11 $n 50]$ which means that the threshold is determined by averaging the intensities over $(2 \times 50+1)^{2}$ pixels in the vicinity area of a particular point. This can be rewritten as [8 $n 50]$ when the grain boundary contrast is brighter than the inner grain, which situation corresponds for an input image to a photographic negative. In both cases, the threshold value thus determined is called dynamic.

The next choice for the threshold setting is [ $0 n 50]$ for normal grain boundary contrast and [3 $n 50$ ] for brighter boundary contrast. In this case the threshold is determined automatically from the intensity histogram and the parameter $n 50$ here is dummy.

Final choice possible is for example [4 $n 74]$ where the threshold is manually set as 74 for normal grain boundary contrast. For the same value of manual threshold, [7n74] is for the case where grain boundary contrast is brighter.

(2) Choosing a Method to Extract Grain Network by an Area Analysis

Default setting is [0 $n 10]$ by which regions of the maximum area are extracted as grain network. If it is rewritten as [ $\left[\begin{array}{ll}1 & n 10\end{array}\right]$, then regions larger than 0.9 times the maximum area are extracted. In both cases, the parameter $n 10$ is dummy.

If [2 $n 4$ ] is chosen the extraction is done for regions larger than the maximum area minus $1 / 4$ of that, whereas if [ $3 \mathrm{nl0}$ ] is chosen it is done for regions larger than 10 pixels in area. The other choice is [4 $n 2]$ or
[4 $n 3$ ] by which regions larger than the second largest or the third largest area are extracted as grain networks.

(3) Excluding Small Areas Having Less than the Number of Pixels Designated

Default value is [20] where the number of pixels is the direct input.

(4) Eliminating Fluffs along Grain Networks

Default value is [4] and an integer between 2 and 7 can be chosen according to the power for the elimination, 2 for being the weakest and 7 for the strongest.

(5) Saving Fluffy but Long Elements as a Part of Grain Network

Default setting is $[3(W) 10(L)]$ which means that a fluffy element longer than $L$ pixels and thinner than $(2 W+1)$ is regarded as a part of a grain boundary.

Note that in the "STARTUP2", only parameters concerning the above terms from (1) through (3) can be adjusted by users.

\subsection{Effect of Varying Parameters on the Construction of a Grain Network}

The "STARTUP" batch file with default parameters on the above items can be rewritten using an appropriate editor. An effort is made to examine how a change in default parameter settings alters the result on construction of the grain network followed by the repair of the grain boundaries. As an input image an optical micrograph of ferrite grains is used. It is one of and is the best in quality of the micrographs being used for the following sections to examine the effect of sample preparation method on the grain size measurement.

Figs. 1(a) and 1(b) show the results of grain boundary thinning and the repair obtained using "STARTUP1" with all the default settings. Total number of grains being automatically displayed after the successful repair is 302 for grains completing their circuit, 77 for those interrupted by the border, and 379 as a total. These characteristic numbers of grain are hereafter referred to as $I, B$ and $T$, respectively. The results in Fig. 1 is taken as a reference in the following examination, where the effect of changing default parameters is discussed in the corresponding order of the items (1) through (5) in Sec. 2.1.

\section{(1) Binarization}

It turns out that binarization of the input image is unsuccessful by any change made to the default setting. For a normal optical micrograph of grains with darker grain boundary contrast, the dynamic threshold under the default condition seems to be the best choice.

\section{(2) Area Analysis}

It was found that the results are the same by the parameter settings for [ $1 n 10],[2 n 4]$ and that they are quite similar for [ 3 n10] and [4 $n 2]$. An appreciable difference can only be seen by a choice between [ $4 n 2]$, being equivalent to the default setting in this case, and [ 4 n3], whose sults after the 

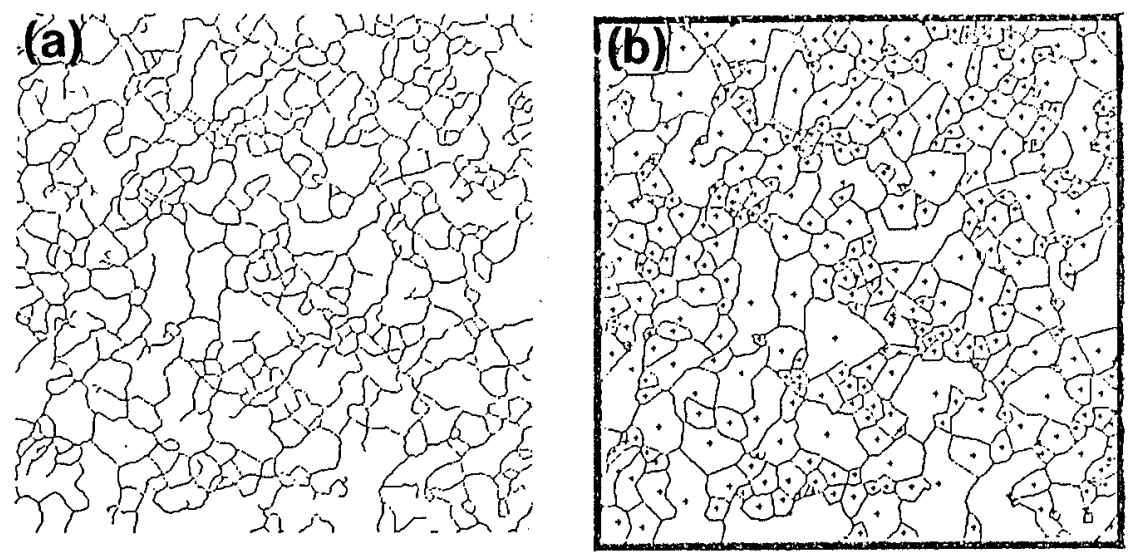

Fig. 1.

Results of (a) grain boundary thinning and (b) the following grain boundary repair for the reference sample which is M7 appearing in Table 2.

Fig. 2.

Difference in the results after the grain boundary repair between by choosing (a) [ $\left.\begin{array}{ll}4 & n 2\end{array}\right]$ and (b) [ $\left.\begin{array}{ll}4 & n 3\end{array}\right]$ for the area analysis on grain network extraction.
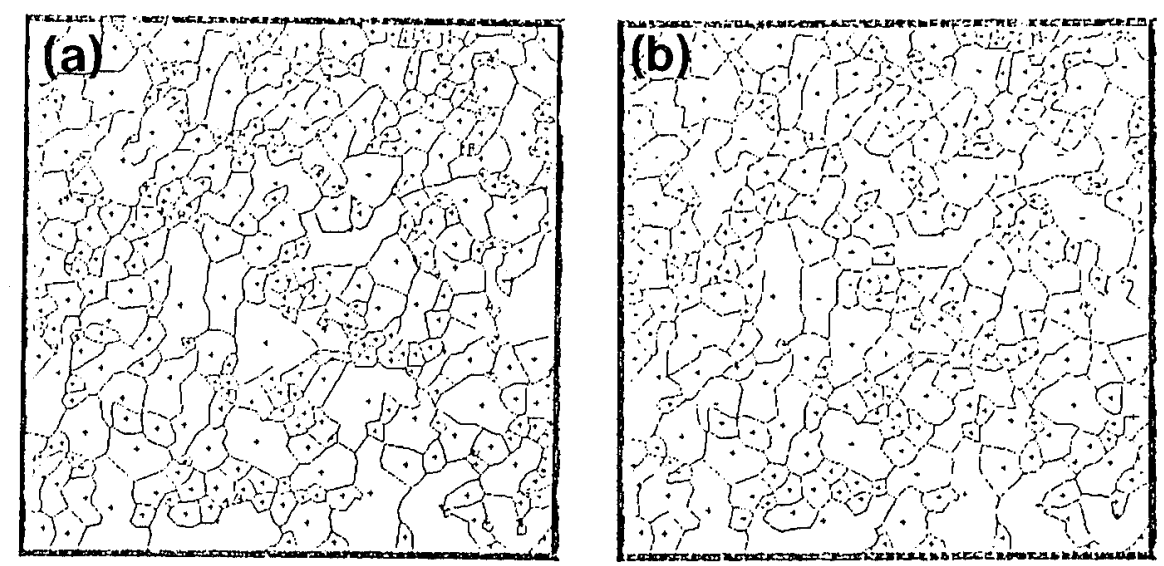

repair process are compared in Fig. 2 with numbers of $I, B$ and $T$ grains. The $I$ and hence $T$ grains are obviously evaluated to be much less for the latter setting.

\section{(3) Excluding Small Areas}

Only a case to put [50] pixels instead of the default [20] is examined here and the result, being $I=222$, $B=67$ and $T=289$, is found to be nearly the same as that for Fig. 2(b).

\section{(4) Elimination of Fluffs}

Two extremes, namely [2] and [7], are chosen instead of default value of being [4]. The results are $I=209, B=67$ and $\mathcal{T}=276$ for the former case and $I=225, B=67$ and $T=292$ for the latter. In both cases, the total number of grains are estimated to be less than that obtained by default setting. To understand this apparent inconsistency, understanding on the details of algorithm for this process should be necessary.

\section{(5) Saving Long Fluffy Elements}

Table 1 shows the effect of combination of $W$ and $L$ on the number of grains obtained after the thinning and repair processes. It seems that the parameter change in $L$ affects the results, particulary on $I$, more than that in $W$ does and that any change hereby examined results in less number of grains as compared to what obtained by the default setting.

Above results indicate that the default settings in the "STARTUP1" are very reasonably made to carry out the grain boundary thinning and repair
Table 1. Effect of changing parameters for saving a fluffy but long element as a part of grain boundary on the measured number of grains.

\begin{tabular}{lcccc}
\hline \multirow{3}{*}{ Grains } & \multicolumn{4}{c}{ Combination of $\left[\begin{array}{ll}W & L\end{array}\right]$} \\
\cline { 2 - 5 } & $\begin{array}{c}\text { Default } \\
{\left[\begin{array}{lll}3 & 10\end{array}\right]}\end{array}$ & {$\left[\begin{array}{ll}3 & 20\end{array}\right]$} & {$\left[\begin{array}{ll}3 & 5\end{array}\right]$} & {$\left[\begin{array}{ll}9 & 5\end{array}\right]$} \\
\hline$B$ (Border) & 77 & 65 & 71 & 71 \\
$I$ (Inside) & 302 & 198 & 235 & 235 \\
$T$ (Total) & 379 & 263 & 306 & 306 \\
\hline
\end{tabular}

processes for the sample micrograph. Any difference that could be resulted by the change in the default parameter is found to be less in number of total grains by up to $30 \%$. It is to be noted that the grain network constructed by hands on the same micrograph used here by several metallurgical engineers is in all cases turned out to be even finer, giving larger number of grains, than that shown in Fig. 1(b). Although the comparison is qualitative, the method developed here with the default settings can be regarded to be quite valid and useful for the current purpose.

Final comment in this section is that the usage of "STARTUP2" is found to be unsuccessful in all cases including the case using the default settings. During the course of the GSM Group activities, it has been pointed out that a reasonable number of grains to be included in the input image within $256 \times 256$ pixels are 100 to $300 .^{3)}$ The grain size of the sample used here is finer than this guideline, which could be the reason for not being able to use the time-saving 
version of the "STARTUP".

\section{Effect of Sample Preparation Method}

The effect of sample preparation method or, in other words, that of input image quality on the result of grain boundary thinning and repair processes is examined. The conditions of the samples are listed in Table 2. The original micrographs and their binarized images are shown in Figs. 3 through 10 . To be noted is that upon examination a common area of single specimen is used under different conditions of preparation. Also note that the sample M7 was used in the previous section.

The results are listed in Table 3 of thinning and repair processes using "STARTUP1" with default settings for M1 through M8. In the table, $O$ indicates the process to be completed successfully, $\Delta$ to be completed but meaninglessly, and $\times$ to be incompleted. It is understandable that both processes are unsuccessful for M1 because the state of polishing is roughest of all. The reason for $\mathrm{M} 2$ to be able to go through the thinning process would be that the sur-

Table 2. Conditions for the sample preparation.

\begin{tabular}{ccl}
\hline $\begin{array}{c}\text { Sample } \\
\text { No. }\end{array}$ & Wet polishing & $\begin{array}{l}\text { State of } \\
\text { etching }\end{array}$ \\
\hline M1 & Up to \#1200 emery paper & $\begin{array}{l}\text { Normal } \\
\text { Over }\end{array}$ \\
M2 & Normal \\
M3 & $7 \mu \mathrm{m}$ diamond paste & Over \\
M4 & " & Normal \\
M5 & $3 \mu \mathrm{m}$ diamond paste & Over \\
M6 & Normal \\
M7 & $1 \mu \mathrm{m}$ diamond paste & Over \\
M8 & $"$ & \\
\hline
\end{tabular}

Fig. 3.

(a) Original input image and (b) corresponding binarized image of sample M1 in Table 2.

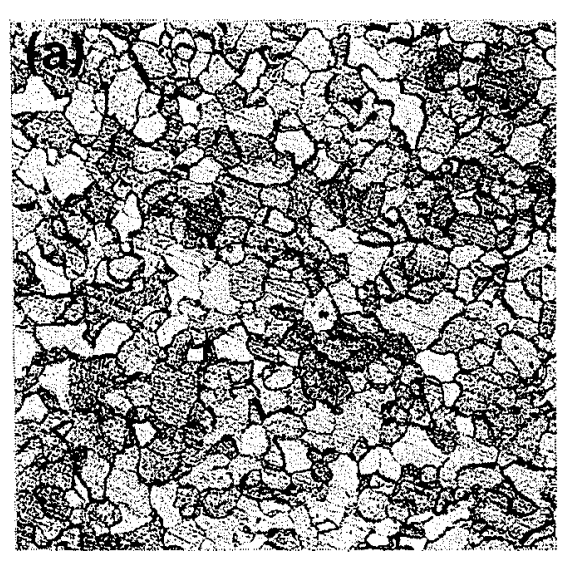

Fig. 5.

(a) Original input image and (b) corresponding binarized image of sample M3 in Table 2.
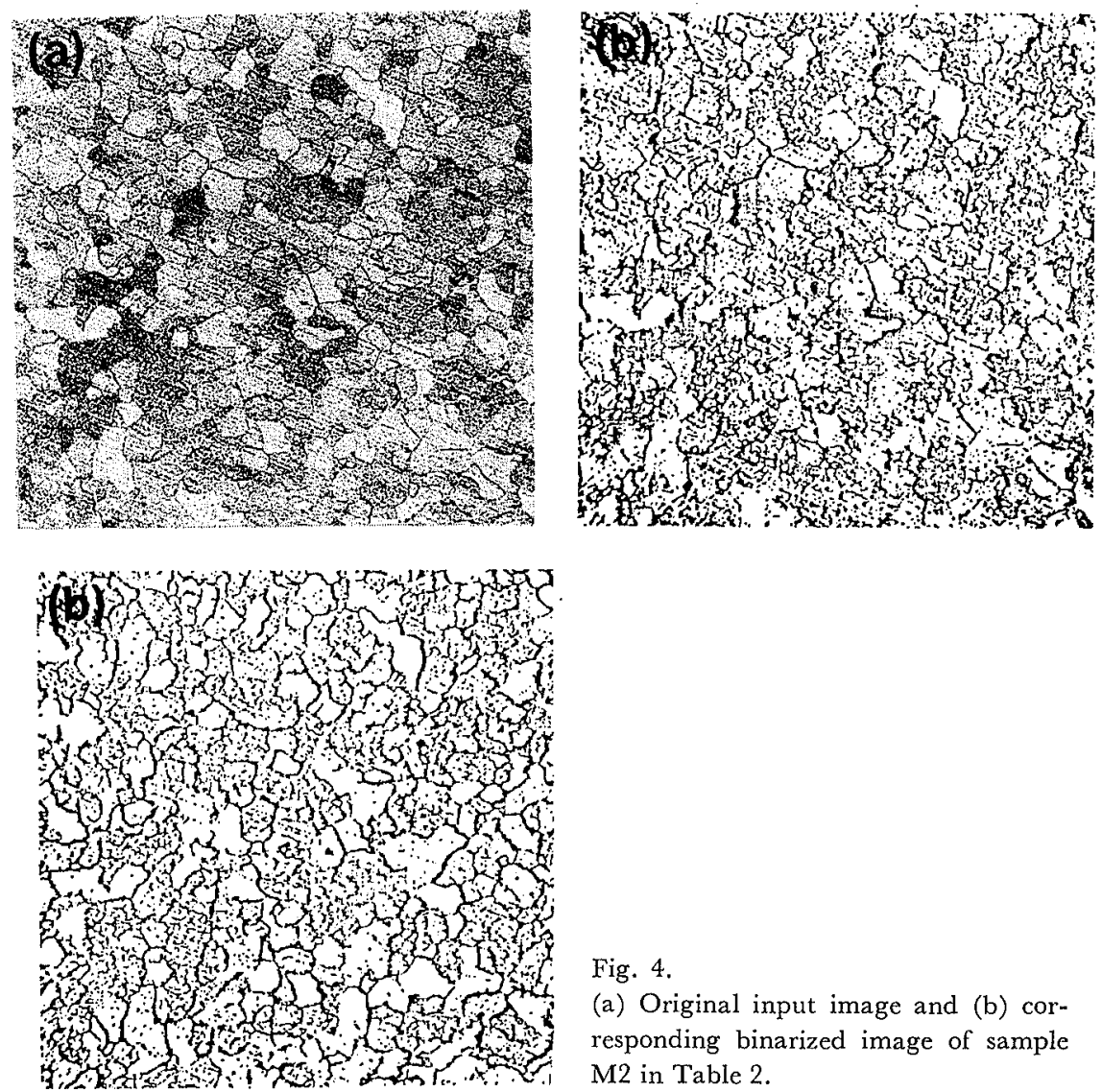

Fig. 4.

(a) Original input image and (b) corresponding binarized image of sample M2 in Table 2.

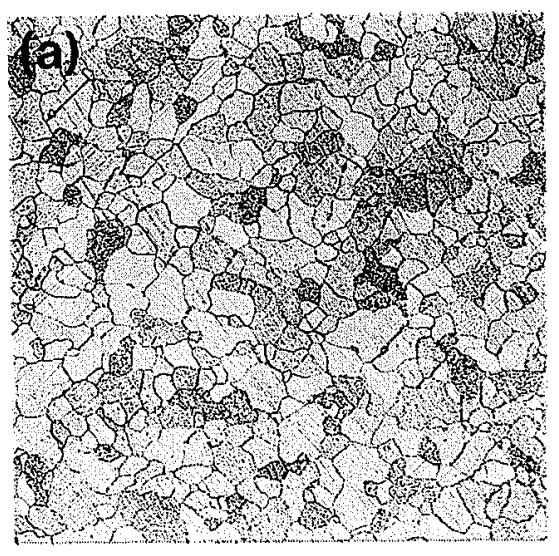

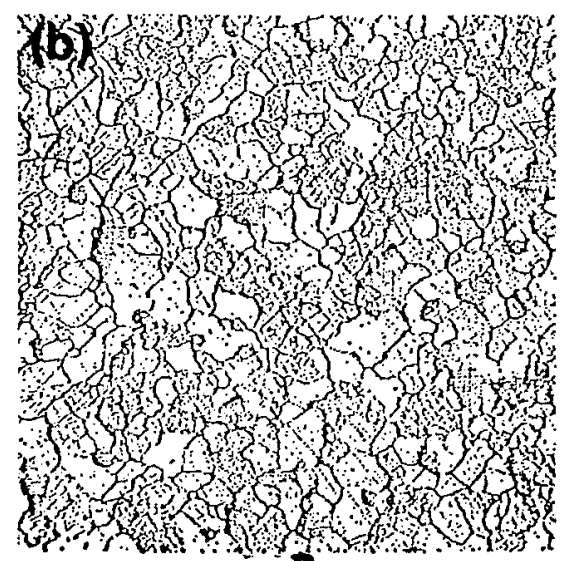



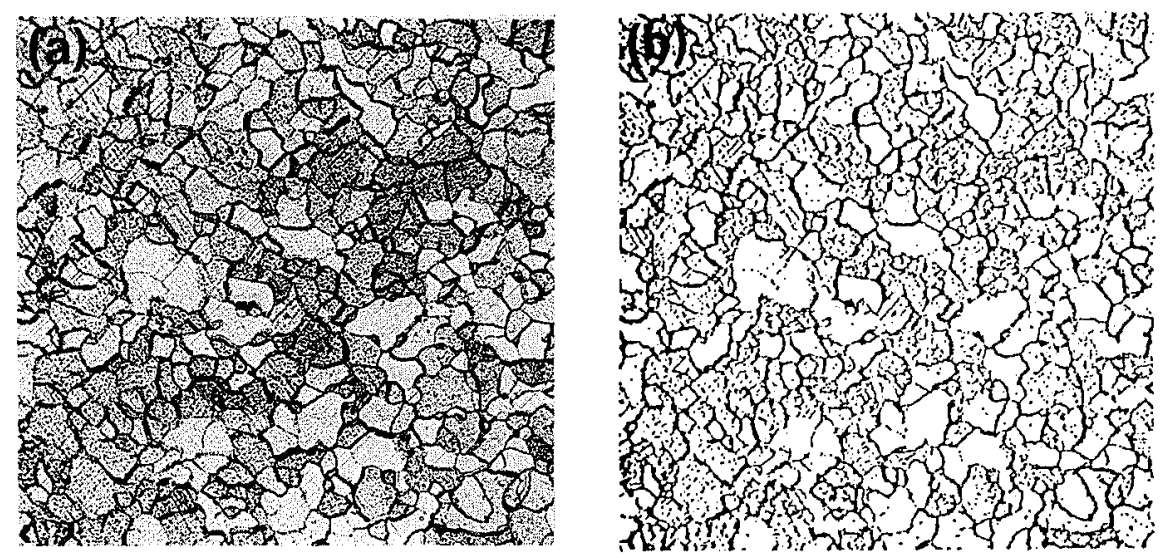

Fig. 6.

(a) Original input image and (b) corresponding binarized image of sample M4 in Table 2.
Fig. 7.

(a) Original input image and (b) corresponding binarized image of sample M5 in Table 2.
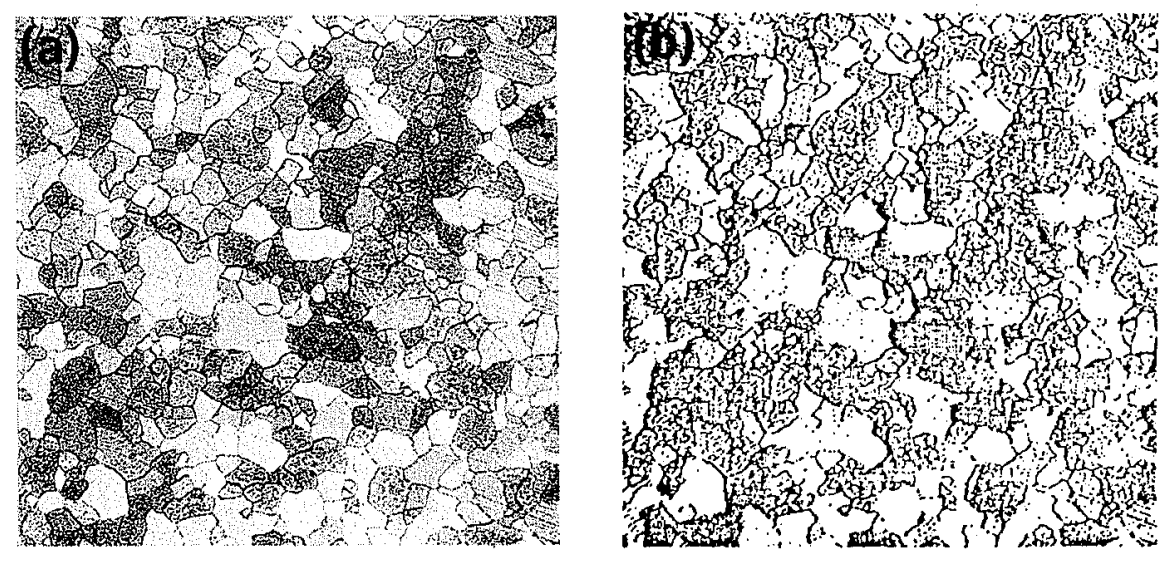
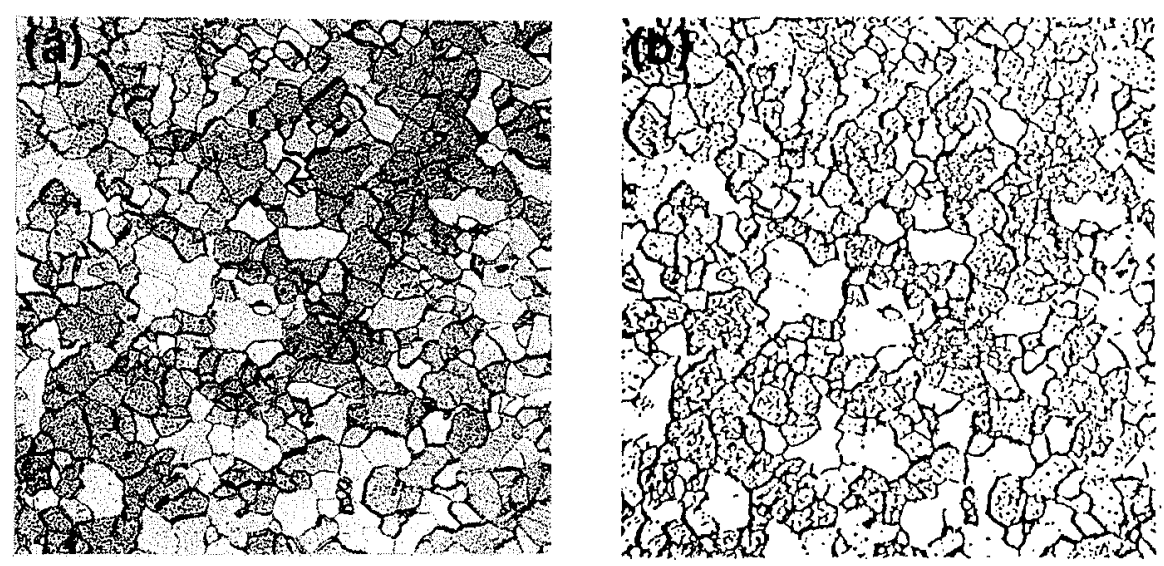

Fig. 8.

(a) Original input image and (b) corresponding binarized image of sample M6 in Table 2.
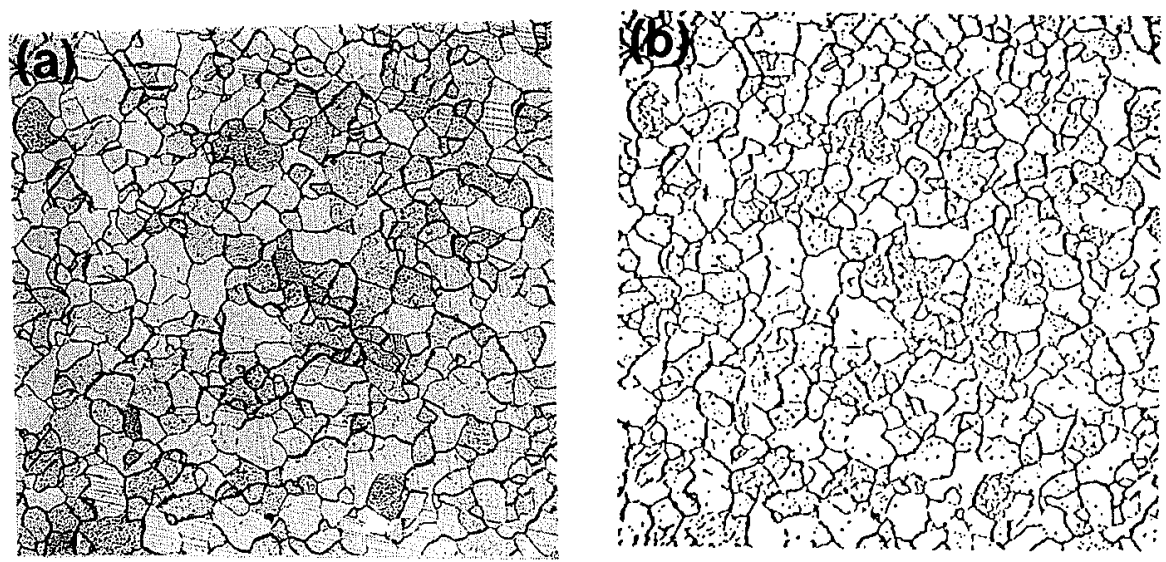

face roughness being the same as M1 might be hindered by the effect of an overetching, however, it does not make any sense because the following repair process is not completed. In M5 again both proc- esses are not successful but in this case, as judged from Fig. 7, it should be because there is a strong contrast difference from grain to grain. An important aspect in this table is that, as far as the etching 
Fig. 10.

(a) Original input image and (b) corresponding binarized image of sample M8 in Table 2.
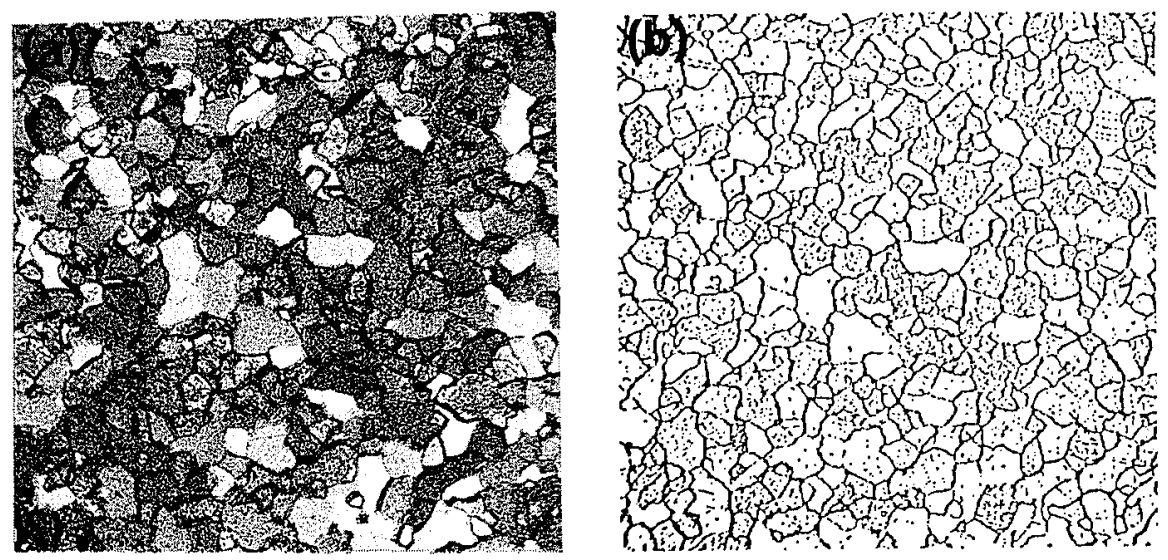
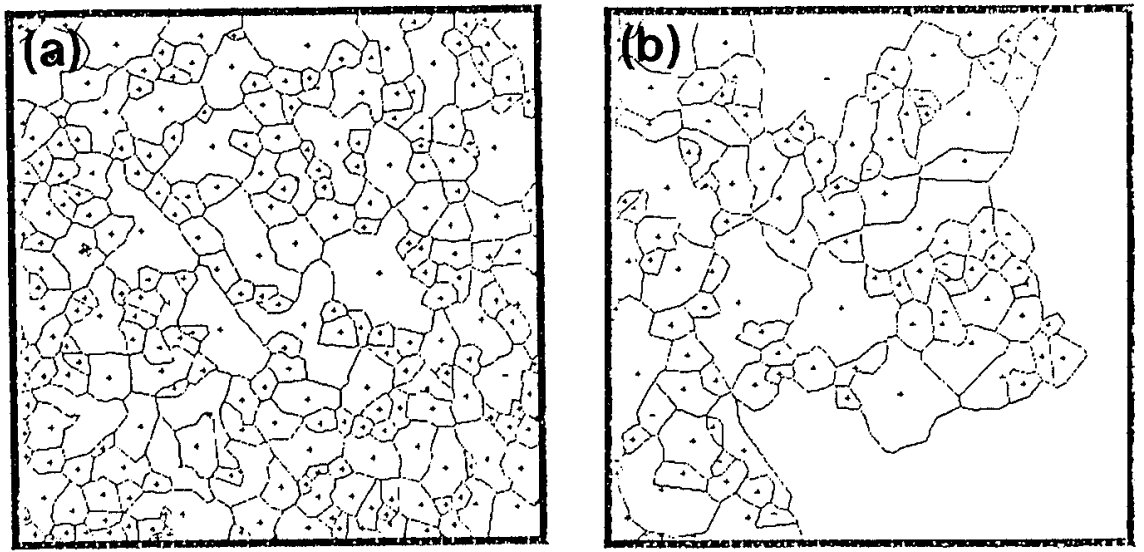

Fig. 11.

Results of grain repair for samples of M2 and $\mathrm{M} 5$ in Table 2 after changing the default parameters. In both cases [4 $n 2$ ] is chosen for area analysis and [50] is chosen for excluding small areas. Numbers of grains obtained are $I=190, B=$ 65 and $T=255$ for $M 2$, and $I=91, B=$ 23 and $T=114$ for M5.
Table 3. Results of grain boundary thinning and repair using "STARTUP1" with default parameters.

\begin{tabular}{|c|c|c|c|c|c|}
\hline \multirow{2}{*}{$\begin{array}{c}\text { Sample } \\
\text { No. }\end{array}$} & \multirow[b]{2}{*}{ Thinning } & \multirow[b]{2}{*}{ Repair } & \multicolumn{3}{|c|}{ Number of grains } \\
\hline & & & $\begin{array}{c}B \\
\text { (Border) }\end{array}$ & $\begin{array}{c}I \\
\text { (Inside) }\end{array}$ & $\begin{array}{c}T \\
\text { (Total) }\end{array}$ \\
\hline M1 & $\Delta$ & $x$ & - & - & - \\
\hline M2 & 0 & $x$ & - & - & - \\
\hline M3 & 0 & 0 & 59 & 245 & 304 \\
\hline M4 & 0 & 0 & 80 & 282 & 362 \\
\hline M5 & $\triangle$ & $x$ & - & - & - \\
\hline M6 & 0 & 0 & 62 & 291 & 353 \\
\hline M7 & 0 & 0 & 77 & 302 & 379 \\
\hline M8 & 0 & 0 & 64 & 316 & 380 \\
\hline
\end{tabular}

$O$ : The process to be successfully completd

$\Delta$ : The process to be completed but meaninglessly

$x$ : The process to be incompleted

is properly done, no significant difference is observed due to the difference in the fineness of the diamond paste used. The difference among the results of M4 through M8 would be acceptable in a practical sense.

An attempt is then made to see whether it is possible or not to obtain a reasonable result by changing the default parameters from a sample for which the thinning or repair process was unsuccessful. Trial and error type effort is therefore made for $\mathrm{M} 1, \mathrm{M} 2$ and M5 using the "STARTUP1" by changing default parameters except for the binarization process which was already shown to work only with the default settings. It is impossible to go through all the possible combinations of parameters, however, two cases are found where M2 and M5 are completed their repair process. The results are shown in Figs. 11 (a) and 11(b). Unfortunately after all the effort, the results are far from reality especially for M5. It is therefore concluded that it is very difficult to save a poorly prepared sample by just changing parameters on these processes. One may think that the software developed here is not very intelligent in this sense, however, it should be stressed that for a reasonably well prepared sample it provides a consistent and reliable answer in about a few tens of minutes.

\section{Limitations Arising from Complexity in Grain Structure}

It has already been mentioned that the purpose of the software developed here by the GSM group is to measure the grain size of a simple grain network. However, it would be necessary for better understandings to show examples of micrographs for which the grain size measurement is impossible with the software. Figs. 12 through 14 are such examples, where Fig. 12 is a microstructure of a medium carbon, manganese steel which consists of ferrite and pearlite two phases, Fig. 13 is that of martensitic structure and Fig. 14 is that of a stainless steel prepared by powder metallurgy. It can be said that the software is not capable of handling such microstructural complexity as decoration at grain boundaries by the second phase, substructures within the grain, and finely dispersed precipitates or inclusions with strang sontrast. 


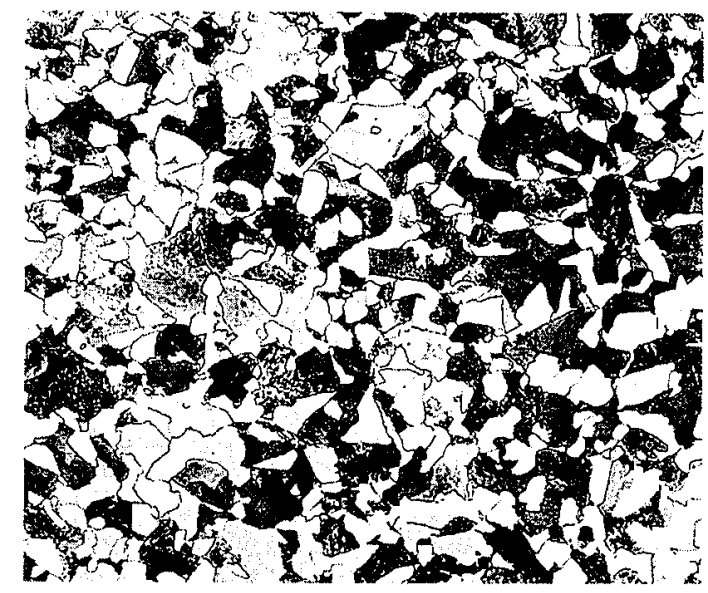

Fig. 12. An optical micrograph of a medium carbon, manganese steel showing ferrite structure with perlite at grain boundaries.

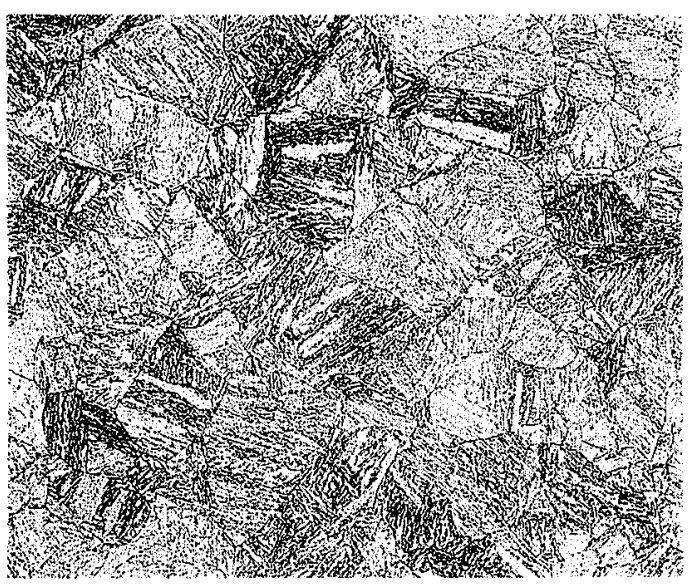

Fig. 13. An optical micrograph of a martensitic structure of a low carbon steel.

\section{Concluding Remarks}

Practical aspect of the software to measure grain size developed by the Grain Size Measurement Group in the Committee on Materials Evaluation by Image Analysis has been discussed. The focus is put on the processes of binarization of the input image, grain boundary thinning and its repair. The effects of change in the default settings for the first two processes are examined on the result of the following repair process. Also the effect of sample preparation

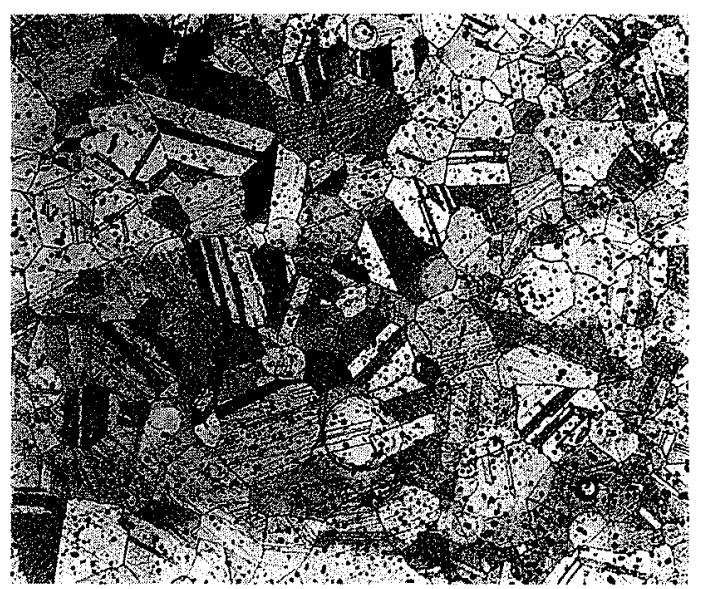

Fig. 14. An optical micrograph of a stainless steel prepared by powder metallurgy.

condition is examined on the result of these processes.

It can be concluded that the grain boundary extraction, thinning and repair processes are successfully done with default parameter settings for specimens of simple grain structures polished to diamond pastes of $3 \mu \mathrm{m}$ or finer before etching, where it is found that the effect of etching condition less affects the results. For input images with less quality, the processes could be completed either with default parameters or by modifying the default settings. However, the results in most cases do not represent the original grain structure and therefore are meaningless.

To be noted is that the input grain structure which can be handled by the present software should be as simple as possible. For input images containing such complexities as grain boundary decoration by second phase, precipitate dispersion and grain substructure cannot go through the grain boundary thinning process.

\section{Acknowledgement}

The author is grateful to Mr. Equo Kobayashi, a graduate student at Tokyo Institute of Technology, for his assistance in sample preparation.

\section{REFERENCES}

1) K. Sakaue: ISIJ Int., 30 (1990), 475.

2) T. Takeuchi: ISIJ Int., 30 (1990), 482.

3) K. Ito: ISIJ Int., 30 (1990), 490. 